\title{
Influência da habilidade comunicacional dos pais nas orientações de saúde ao filho surdo
}

\author{
Influence of parents' communication skills on health guidelines for deaf children \\ Influencia de las habilidades de comunicación de los padres en las pautas de salud para niños sordos
}

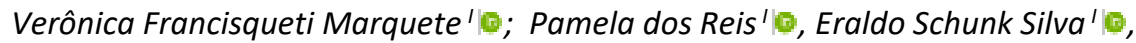

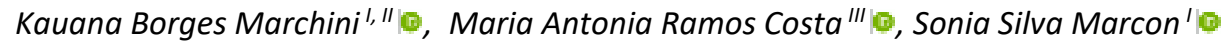

'Universidade Estadual de Maringá, Maringá, PR, Brasil; "Universidade Estadual de Londrina, Londrina, PR, Brasil; 'I'Universidade Estadual do Paraná, Curitiba, PR, Brasil

\begin{abstract}
RESUMO
Objetivo: verificar a influência da comunicação dos pais no recebimento de informações e comportamento de saúde de filhos surdos. Método: estudo transversal realizado com 110 pessoas surdas, selecionadas com a técnica snowball sampling, em municípios no Noroeste do estado do Paraná. Os dados foram coletados de fevereiro a agosto de 2019, mediante aplicação de instrumento estruturado. Na análise dos dados utilizou-se a estatística descritiva e inferencial. Resultados: a maioria dos surdos $(95,4 \%)$ recebeu alguma orientação de saúde no núcleo familiar; ser orientado sobre a importância de consultas de rotina mostrou-se associado à realização da mesma pelos filhos no último ano $(\mathrm{OR}=3,40)$. $O$ pai que sabe se comunicar em Libras constituiu fator de proteção, pois, nestes casos, ocorreram mais orientações sobre o uso de drogas e consumo abusivo de açúcar. Conclusão: a habilidade comunicacional dos pais possibilita que eles realizem mais orientações relacionadas à saúde e isto influencia o comportamento em saúde de filhos surdos.
\end{abstract}

Descritores: Surdez; Família; Relações Familiares; Promoção da Saúde; Comunicação.

\begin{abstract}
Objective: to ascertain the influence of parents' communication on deaf children's reception of health information, and health behavior. Method: in this cross-sectional study of 110 deaf people selected by snowball sampling in municipalities in northwest Paraná state, data were collected from February to August 2019 using a structured instrument. Descriptive and inferential statistics were used in the data analysis. Results: most deaf people (95.4\%) received some health guidance in the family; being guided about the importance of routine appointments was found to be associated with the children's attending such appointments in the prior year $(O R=3.40)$. The parent able to communicate in Libras was a protective factor, because in these cases, more guidance on drug use and sugar abuse occurred. Conclusion: the parents' communication skills enabled them to provide more health-related guidance, and this influenced their deaf children's health behavior.
\end{abstract}

Descriptors: Deafness; Family; Family Relations; Health Promotion; Communication.

\section{RESUMEN}

Objetivo: determinar la influencia de la comunicación de los padres en la recepción de la información sanitaria y el comportamiento sanitario de los niños sordos. Método: en este estudio transversal de 110 personas sordas seleccionadas por muestreo de bola de nieve en municipios del noroeste del estado de Paraná, los datos se recolectaron de febrero a agosto de 2019 mediante un instrumento estructurado. Se utilizó estadística descriptiva e inferencial en el análisis de datos. Resultados: la mayoría de las personas sordas $(95,4 \%)$ recibió alguna orientación sanitaria en la familia; Se descubrió que el hecho de recibir orientación sobre la importancia de las citas de rutina estaba asociado con la asistencia de los niños a dichas citas el año anterior $(\mathrm{OR}=3,40)$. El padre capaz de comunicarse en Libra fue un factor de protección, porque en estos casos, hubo más orientación sobre el uso de drogas y el abuso de azúcar. Conclusión: las habilidades de comunicación de los padres les permitieron brindar más orientación relacionada con la salud, y esto influyó en el comportamiento de salud de sus hijos sordos.

Descriptores: Sordera; Familia; Relaciones Familiares; Promoción de la Salud; Comunicación.

\section{INTRODUÇÃO}

A comunicação é fundamental para o convívio em sociedade, pois possibilita as interações sociais cotidianas e o relacionamento interpessoal ${ }^{1}$. Nesse contexto, a surdez pode constituir importante barreira para as interações sociais, mas pode ser minimizada quando a língua de sinais é utilizada na comunicação².

De acordo com a literatura, a maioria das crianças surdas nasce em famílias ouvintes, que nunca tiveram nenhum tipo de contato com pessoa surda e que desconhecem a língua de sinais ${ }^{3}$, sendo fundamental a escolha e a utilização de uma habilidade comunicacional que seja comum no núcleo familiar. À medida que cresce, a criança precisa adquirir habilidades comunicacionais muito além daquelas utilizadas no seio familiar, e, nesse momento, o mais indicado é a língua de sinais, a qual lhe possibilita a interação com seus pares ${ }^{4}$

Agradecimento à Coordenação de Aperfeiçoamento de Pessoal de Nível Superior - Brasil (CAPES) - Programa de Demanda Social, Mestrado - Código de Financiamento 88882.449135/2019-01. Autora correspondente: Verônica Francisqueti Marquete. E-mail: veronicafrancisqueti@hotmail.com

Editora responsável: Sonia Acioli 
Alguns pais de pessoas surdas, porém, apresentam muita dificuldade e consideram frustrante o fato de terem que aprender a língua de sinais para se comunicarem com seus filhos ${ }^{3}$. Outros consideram não ser possível utilizar a linguagem oral com seu familiar, e, por isto, optam por empregar uma combinação de sinais ou gestos com a escrita ${ }^{5}$.

A parentalidade eficaz é substancial para que ocorra o desenvolvimento intelectual, social, físico e emocional de uma pessoa. As dimensões parentais podem influenciar a personalidade de uma criança surda no futuro e refletir na baixa ou alta autoestima em outras etapas da vida. Portanto, crianças surdas podem tornar-se adultos bem-sucedidos com a ajuda de seus pais 6 .

Isto também é aplicável em relação aos comportamentos em saúde. Ou seja, os pais de pessoas surdas têm grande responsabilidade sobre a condição de saúde de seus filhos, não somente enquanto são crianças, pois, de modo geral, não existe equidade no acesso dessas pessoas a informações relacionadas à saúde. Mesmo existindo leis e decretos, no ordenamento jurídico brasileiro, que asseguram aos surdos a prestação de serviços de saúde que atendam suas necessidades comunicacionais, eles não são suficientes para eliminar as barreiras de comunicação e garantir que a mesma ocorra com qualidade ${ }^{7}$.

Nos estabelecimentos de saúde, normalmente não existem intérpretes, e a maioria dos profissionais de saúde não sabe se comunicar com pessoas surdas. Por estas razões, a população surda tem mais dificuldade de acesso às ações de promoção da saúde e prevenção de doenças. Além disso, as campanhas de saúde, na maioria das vezes, não são disponibilizadas em língua de sinais ou em formatos acessíveis para esse público ${ }^{8}$. Esses aspectos, isolados ou associados, podem contribuir para que as barreiras de comunicação existentes no núcleo familiar dificultem ainda mais o acesso das pessoas surdas às orientações de saúde.

Diante desse contexto, questiona-se: as habilidades comunicacionais dos pais possibilitam que seus filhos surdos tenham mais acesso a informações de saúde? Para responder a este questionamento definiu-se como objetivo do estudo: verificar a influência da comunicação dos pais no recebimento de informações e comportamento de saúde de filhos surdos.

\section{REVISÃO DA LITERATURA}

A promoção da saúde é uma estratégia das políticas públicas direcionada ao atendimento individual e coletivo, visando a autonomia, qualidade de vida e incentivo ao autocuidado ${ }^{9}$. Porém, esta política pública não atinge a população surda com equidade, o que torna esse público mais suscetível ao desenvolvimento de problemas de saúde evitáveis, e também leva a complicações das condições crônicas, devido ao retardo ou subdiagnóstico e subtratamento das mesmas $^{10}$.

A comunicação na Língua brasileira de sinais (Libras) pelo familiar da pessoa surda é substancial para que a educação, orientação, cuidados com a saúde e troca de experiências ocorram de forma adequada. Quando os familiares não sabem se comunicar em Libras, apresentam mais insegurança em repassar informações e orientações relacionadas à promoção da saúde e prevenção de doenças, repercutindo em um conhecimento limitado dos surdos sobre o processo saúde doença ${ }^{11}$.

As habilidades comunicacionais empregadas pelas pessoas surdas também influenciam o atendimento prestado nos serviços de saúde. Surdos que utilizam apenas a língua de sinais têm mais chances de não entender o seu diagnóstico, enquanto os oralizados ou bilíngues têm mais chances de compreender o profissional de saúde, o seu tratamento e diagnóstico. Estratégias de comunicação utilizadas pelos profissionais, como o uso da oralização e da língua portuguesa escrita, foram apontadas pelas pessoas surdas que se comunicam apenas por sinais como as mais prejudiciais na interação entre profissionais de saúde e surdos ${ }^{12}$.

Quando comparadas com as pessoas ouvintes, percebe-se que as pessoas surdas empregam com maior frequência comportamentos de risco para a saúde: sedentarismo, obesidade e não realização do tratamento adequado para doenças cardiovasculares ${ }^{10}$. Assim, destaca-se a importância de esse público ter uma assistência à saúde inclusiva, com qualidade e equidade, para que ocorram o autocuidado, a promoção, recuperação e reabilitação da saúde ${ }^{13}$.

\section{MÉTODO}

Estudo transversal, desenvolvido com pessoas surdas residentes em municípios afetos ao Arranjo Populacional Principal (AAP) da região metropolitana de Maringá (RMM), na região Noroeste do estado do Paraná ${ }^{14}$.

Para localização dos possíveis participantes, foi contactada a associação de surdos da cidade, considerando-se que os surdos constituem uma população de difícil acesso e mensuração. Contudo, a instituição realiza apenas atendimentos por demanda espontânea, com atividades programadas esporadicamente. Sendo assim, optou-se por utilizar a técnica de amostragem não probabilística, snowball sampling, que pressupõe que indivíduos com características comuns têm 
laços e estão conectados a uma rede social ${ }^{15}$, e, por isto mesmo, tendem a ser identificados com maior facilidade por outros membros de sua rede social do que pelos pesquisadores ${ }^{16}$.

Os critérios de inclusão adotados foram: ter 18 anos ou mais, com perda auditiva profunda desde a infância e saber se comunicar em Libras. Por sua vez, o único critério de exclusão adotado foi a presença de algum distúrbio cognitivo (referido por familiares próximos), o que ocorreu em dois casos.

O primeiro recrutamento ocorreu durante participação intencional da pesquisadora principal em uma palestra referida associação, na qual estiveram presentes oito pessoas surdas, das quais duas aceitaram participar da pesquisa e indicaram outras cinco pessoas, que, provavelmente, poderiam concordar em participar. Simultaneamente, foi iniciada, na rede social Facebook, a busca por pessoas que, em seu perfil, se identificassem como surdas e residentes nos municípios em estudo, sendo-Ihes enviado convite "in box". Em caso de aceite, foi definido dia, hora e local mais convenientes ao participante para a coleta de dados, que ocorreu em espaços públicos de fácil acesso, domicílios e estabelecimentos comerciais (shoppings e supermercados).

Ressalta-se que ao final de cada encontro solicitou-se a indicação de outros possíveis participantes e assim sucessivamente, até o momento em que todos os membros acessíveis já tinham sido contactados e as indicações se tornaram repetitivas ${ }^{15}$. No total, 263 pessoas foram-convidadas a participar do estudo, sendo que 151 não aceitaram e duas foram excluídas. Deste modo, a amostra estudada foi constituída de 110 pessoas.

Os dados foram coletados no período de fevereiro a agosto de 2019 , mediante aplicação de instrumento pela pesquisadora principal, que tem capacitação em Libras. O instrumento utilizado na coleta de dados, do tipo estruturado, continha quatro partes. A primeira abordava características sociodemográficas: idade, sexo, raça, escolaridade, trabalho renumerado, estado civil, companheiro surdo e uso de órteses. A segunda parte era constituída por variáveis relacionadas à comunicação: idade em que começou a comunicar-se em Libras; habilidade em fazer leitura labial; utilização de comunicação oralizada; percepção da comunicação familiar; compreensão da Língua portuguesa; se comunica por escrito com quem não sabe Libras; mãe se comunica em Libras e pai se comunica em Libras. A terceira parte referia-se às orientações familiares relacionadas aos hábitos alimentares, com respostas dicotômicas. Por fim, a quarta parte continha questões sobre o estilo de vida, também com respostas dicotômicas.

Os dados foram digitados e armazenados em planilha Excel ${ }^{\oplus}$ e analisados com auxílio da estatística descritiva e inferencial no software SAS (Statistical Analysis Software), versão 9.4. Considerou-se, como medida de associação, a razão de chance (Odds Ratio), com intervalo de confiança de $95 \%$, sendo calculada a partir do ajuste do modelo de regressão logística.

O projeto do estudo foi aprovado pelo comitê de ética em pesquisa com seres humanos da instituição signatária (Parecer n.o 2.959.112) e todos os participantes assinaram o Termo de Consentimento Livre e Esclarecido (TCLE) em duas vias.

\section{RESUltados}

As 110 pessoas surdas em estudo tinham idade entre 18 e 73 anos (média de 35 anos $\pm 11,5$ ), pouco mais da metade era do sexo feminino (51,8\%), da raça branca (62,7\%), 45,5\% tinha Ensino Médio completo, 69,1\% estava inserida no mercado de trabalho, 70,0\% tinha companheiro, sendo que $61,7 \%$ destes o parceiro também era surdo. Quanto à surdez, 44,5\% relatou que o diagnóstico ocorreu logo após o nascimento, e para os demais, em torno de um ano $(20,9 \%)$, dois anos $(14,5 \%)$, três anos $(8,2 \%)$, quatro anos $(5,4 \%)$ e dos cinco aos 12 anos $(6,4 \%)$. A idade em que começaram a se comunicar em Libras variou de um a 42 anos, sendo a média de 9,9 anos.

Observou-se que em 18,3\% dos casos os pais se comunicam em Libras, e 44,9\% apenas as mães utilizam esta modalidade linguística. E filhos do sexo feminino tiveram quatro vezes mais chances $(O R=4,22 ; \mathrm{IC} 95 \%$ : $1,41-12,62 ; \mathrm{p}$ valor $=0,0101$ ) de o pai não saber se comunicar em Libras.

Além da Libras, os participantes também relataram utilizar outras habilidades comunicacionais, sendo que $46,4 \%$ utilizava a leitura labial (outros $40,9 \%$ às vezes consegue), $34,5 \%$ possuíam comunicação oralizada (outros $39,1 \%$ às vezes consegue); e 16,5\% compreendia a língua portuguesa escrita (outros $66,1 \%$ às vezes consegue). Constatou-se que $41,28 \%$ consideravam boa a comunicação familiar; 31,2\%, péssima/ruim/regular, e 27,5\%, ótima.

No que tange às orientações de saúde no núcleo familiar, constatou-se que a maioria $(95,4 \%)$ referiu receber algum tipo de orientação (Tabela 1), e que filhos cujos pais orientam sobre a importância das consultas de rotina apresentaram três vezes mais chances de as terem realizado no último ano (OR=3,40; IC 95\%: 1,53-7,55; $p$ - valor= 0,0042). 
TABELA 1: Orientações de saúde no núcleo familiar de pessoas surdas. Municípios com arranjo populacional principal da região metropolitana de Maringá, PR, Brasil, 2019.

\begin{tabular}{lll}
\hline A família realiza orientações: & $\mathbf{n}$ & \% \\
\hline Importância do consumo de água & 98 & 89,9 \\
Importância de fazer exercícios físicos & 70 & 64,2 \\
Evitar consumo de frituras & 70 & 64,2 \\
Perigos do consumo excessivo de açúcar & 69 & 63,3 \\
Perigos do consumo excessivo de sal & 68 & 62,4 \\
Importância de consultas de rotina & 60 & 55,0 \\
Não utilizar drogas & 57 & 52,3 \\
\hline
\end{tabular}

Identificou-se ainda que filhos de mães $(O R=3,00$; IC 95\%=1,28- 6,99; $p$-valor=0,0112) e pais $(O R=2,87$; IC $95 \%=$ $1,06-7,75 ; p$-valor $=0,0382$ ) que não sabem se comunicar em Libras têm mais chances de sentirem-se nervosos e estressados. (Dados não apresentados em tabela).

A Tabela 2 mostra que o pai que sabe se comunicar em Libras constitui fator de proteção para as orientações sobre drogas e consumo abusivo de açúcar.

TABELA 2: Associação da comunicação em Libras pelo pai de pessoas surdas ao acesso às orientações de saúde sobre os perigos do uso de drogas e do consumo excessivo de açúcar. Municípios com arranjo populacional principal da região metropolitana de Maringá, PR, Brasil, 2019.

\begin{tabular}{|c|c|c|c|c|c|c|c|}
\hline \multirow{3}{*}{ Variáveis } & \multicolumn{4}{|c|}{ Pai se comunica em Libras } & \multirow{3}{*}{$\| p$-valor } & \multirow{3}{*}{ "OR } & \multirow{3}{*}{ IC $(95 \%)^{\S}$} \\
\hline & \multicolumn{2}{|c|}{ Não } & \multicolumn{2}{|c|}{ Sim } & & & \\
\hline & $\mathbf{n}$ & $\%$ & $\mathbf{n}$ & $\%$ & & & \\
\hline \multicolumn{8}{|c|}{ Perigos do uso de drogas Família orienta } \\
\hline Não & 52 & 58,4 & 5 & 25,0 & - & 1 & - \\
\hline Sim & 37 & 41,6 & 15 & 75,0 & 0,0101 & 0,237 & $0,079-0,710$ \\
\hline \multicolumn{8}{|c|}{ Perigos do consumo excessivo do açúcar } \\
\hline \multicolumn{8}{|c|}{ Família orienta } \\
\hline Não & 38 & 42,7 & 2 & 10,0 & - & 1 & - \\
\hline Sim & 51 & 57,3 & 18 & 90,0 & 0,0141 & 0,149 & $0,033-0,682$ \\
\hline
\end{tabular}

*OR = Odds ratio; IC= 95\% Intervalo de confiança; "p-valor = Probabilidade de significância.

\section{DISCUSSÃO}

O fato de a quase totalidade dos participantes afirmar que receberam algum tipo de informação sobre saúde por parte do núcleo familiar certamente está relacionado às suas habilidades comunicacionais, pois a grande maioria consegue utilizar mais de uma forma de comunicação. Nesse tocante, pesquisa realizada na África do Sul revelou que o uso de diferentes modalidades de comunicação ocorria devido à necessidade de compreensão dos familiares. Assim, gestos familiares próprios eram utilizados nos domicílios em que as famílias das pessoas surdas tinham pouco ou nenhum conhecimento da língua de sinais. Esses gestos possibilitavam as interações para que o familiar surdo não ficasse isolado; entretanto, a qualidade da comunicação era prejudicada devido às limitações do gestual informal empregado, falhas neste e a consequente superficialidade na interação ${ }^{3}$. Essas características também devem estar presentes nas famílias de uma parcela dos participantes deste estudo, pois $31,2 \%$ consideram péssima/ruim/regular a comunicação familiar.

A comunicação no núcleo familiar é substancial por possibilitar a construção de vínculos, a transmissão de crenças e valores e ensinar a enfrentar os desafios da vida. A conexão entre as diferentes gerações pode contribuir para o desenvolvimento da resiliência em todas as fases da vida ${ }^{17}$. Estudo realizado em Massachusetts (EUA) identificou que a ausência de uma comunicação acessível com os pais ouvintes é um trauma comum vivenciado durante a infância por adultos surdos ${ }^{18}$.

Observou-se que quando os pais não se comunicam em Libras, os surdos têm mais chances de se sentirem nervosos e estressados. Isto provavelmente ocorre porque a resposta aguda ao estresse, principalmente se for repetida e crônica, pode prejudicar o sistema psicológico e o fisiológico, provocando inflamação estéril (ausência de microorganismos) e a síndrome da resposta inflamatória sistêmica ${ }^{19}$. Assim, a utilização da Língua de sinais pelos familiares, além de auxiliar a relação e a interação familiar, evita a exacerbação de doença infamatória e dor, desencadeadas devido às respostas inflamatórias estéreis ${ }^{20}$. Portanto, é primordial o desenvolvimento de estratégias que orientem e 
sensibilizem os familiares sobre a importância de utilizarem, no seio familiar, uma comunicação adequada o mais precocemente possível.

Ademais, é possível inferir que os familiares interferem no comportamento dos surdos em relação à saúde. Por exemplo, embora apenas um pouco mais da metade dos participantes tenha sido orientado por seus familiares a realizar consultas de rotina, os que as fizeram tiveram significativamente mais chances de fazer consultas periódicas. Nesse sentido, estudo realizado com dados da Pesquisa de Saúde de Rochester para Surdos, de 2013, constatou como possível fator responsável pelas piores condições de saúde dos surdos, a qualidade auditiva dos pais, intermediada pela opção do método de comunicação empregado para com o filho surdo ${ }^{21}$.

As orientações indiretas da família durante a infância, mediante a contação de histórias médicas e discussões gerais sobre saúde da família, constituem importante fonte de conhecimento sobre saúde, domínio em que muitos adolescentes e adultos surdos apresentam lacunas ${ }^{22}$. A orientação familiar indireta na infância é um componente central para oportunidades de aprendizado contextual que influenciam os resultados da saúde do adulto. Nesse contexto, os pais de crianças surdas têm papel fundamental na redução das atuais disparidades de saúde observadas na população surda ${ }^{21}$.

Quando comparadas com os pais, as mães têm maior empenho em se comunicar com os filhos em Libras, independente do sexo. Contudo, quando o filho é do sexo masculino, o pai tem mais chances de se comunicar em Libras, o que favorece a transmissão de informações sobre saúde no âmbito familiar, pois são mais pessoas que podem colaborar nesta tarefa. Nesse contexto, constata-se a predominância do modelo de família patriarcal, em que o pai delega a educação dos filhos à mulher, em especial a educação das filhas, considerada responsabilidade privativa de suas mães ${ }^{23}$. Essa barreira da paternidade e da comunicação com os filhos se torna ainda mais acentuada no contexto que envolve as pessoas surdas. Isto porque, no presente estudo, constatou-se a associação estatisticamente significativa do pai orientar sobre consumo de açúcar e de drogas, sendo que as filhas surdas têm mais chances de não receberem essas orientações.

Esses resultados mostram a importância de o setor saúde trabalhar com pais de crianças surdas no sentido de sensibilizá-los sobre o papel que eles têm na orientação dos filhos e o quanto esta tarefa poderá ser facilitada se conseguirem se comunicar em Libras. Muitas vezes, os pais desconhecem o quanto podem interferir nos comportamentos de saúde dos seus filhos, por meio de orientações e apoio para que os mesmos adotem comportamentos saudáveis em relação à alimentação, prática de atividades físicas, sono e tempo de lazer ${ }^{24}$.

Estudo realizado com adolescentes ouvintes nos Estados Unidos constatou que o elevado/bom funcionamento familiar influencia a adoção de comportamentos preventivos para as doenças crônicas não transmissíveis, pois os adolescentes apresentaram menores chances de sobrepeso, obesidade e distúrbios alimentares. Ressalta-se que no presente estudo também foi observado que o bom relacionamento com os pais pode ter maior impacto nos filhos do sexo masculino ${ }^{24}$

De modo geral, os resultados deste estudo mostram que os profissionais de saúde precisam: desenvolver ações de educação em saúde junto aos pais de crianças surdas, em um ambiente participativo e socializador ${ }^{25}$; reconhecer que a família constitui o locus mais importante para a realização de orientações de saúde, mudanças de hábitos e adoção de comportamentos seguros de saúde. Isso é fundamental para a redução da morbimortalidade, prevenção de doenças, diagnóstico precoce e tratamento oportuno.

\section{Limitações do estudo}

Embora o estudo apresente como limitação o uso da técnica snowball sampling, que impossibilita generalizações sobre os resultados obtidos, destaca-se que esta é substancial para conhecer pessoas ocultas e de difícil acesso. De qualquer modo, os resultados podem subsidiar o desenvolvimento de estratégias que propiciem maior conscientização dos pais de pessoas surdas sobre a importância de sua habilidade de comunicação para que os filhos tenham mais acesso a informações de saúde e possam adotar comportamentos mais seguros e conscientes.

\section{CONCLUSÃO}

Os resultados do estudo mostraram que os comportamentos de saúde das pessoas surdas são influenciados por orientações que elas recebem dos pais, e o emprego da comunicação em Libras proporciona maiores chances de os filhos serem orientados sobre os perigos do uso de drogas e do consumo abusivo de álcool, e também menores chances de sentirem-se nervosos e estressados.

\section{REFERÊNCIAS}

1. Vieira SS, Dupas G, Chiari M. Effects of cochlear implantation on adulthood. CoDAS [Internet], 2018 [cited 2018 Sep 15]; 30(6):e20180001. DOI: http://dx.doi.org/10.1590/2317-1782/20182018001 
2. World Health Organization. Deafness and hearing loss, 2018. Genebra: World Health Organization [Internet], 2018 [cited 2019 Feb 10]. Available from: www.who.int/mediacentre/factsheets/fs300/en/

3. Blose ZM, Joseph LN. The reality of every day communication for a deaf child using sign language in a developing country. Afr. Health Sci. [Internet], 2017 [cited 2020 Jan 26]; 17(4): 1149-59. DOI: https://doi.org/10.4314/ahs.v17i4.24

4. Yamashiro JA, Lacerda CBF. Being the Sibling of a Deaf Person: Reports from Childhood to Adulthood. Rev. Bras. Educ. Espec. [Internet], 2016 [cited 2018 Dec 02]; 22(3):367-80. DOI: http://dx.doi.org/10.1590/S1413-65382216000300005

5. Zaidman-Zait A, Curle D. Complexity: An Interpretative Phenomenological Analysis of the Experiences of Mothers of Deaf Children with Cochlear Implants and ASD. J. Health Serv. Psychol. [Internet], 2016 [cited 2019 Feb 20]; 23(9):1173-84. DOI: http://dx.doi.org/10.1177/1359105316646171

6. Ekim A, Ocakci AF. A Comparison of parenting dimensions between deaf and hearing children. Clin. Nurs. Res. [Internet], 2016 [cited 2019 Mar 10]; 25(3):342-54. DOI: http://dx.doi.org/10.1177/1054773815619133

7. Silva MAMD, Benito LAO. The knowledge of nursing students about brazilian sign language (BSL). Univ. Ciênc. Saúde [Internet], 2016 [cited 2020 Apr 06]; 14(1):23- 30. Available from:

https://www.publicacoesacademicas.uniceub.br/cienciasaude/article/view/3534/3067

8. Mboua CP, Touko A. Psychosocial vulnerability and HIV/AIDS epidemiological situation among people with hearing disabilities in four towns in Cameroon. Med. Sante Trop. [Internet], 2016 [cited 2019 Jun 05]; 26(4):391-95. DOI: http://dx.doi.org/10.1684/mst.2016.0633

9. Janini JP, Bessler D, Vargas AB. Health education and health promotion: impact on quality of life of elderly. Saúde em Debate [Internet], 2015 [cited 2019 Aug 03]; 39(105). DOI: https://doi.org/10.1590/0103-110420151050002015

10. Emond A, Ridd M, Sutherland H, Allsop L, Alexander A, Kyle J. The current health of the signing deaf community in the UK compared with the general population: a cross-sectional study. BMJ Open [Internet], 2015 [cited 2019 Aug 19]; 5(1):e006668. DOI: http://dx.doi.org/10.1136/bmjopen-2014-006668

11. Marquete VF, Teston EF, Souza RR, Vieira VCL, Fischer MMJB, Marcon SS. Desafios do cuidado a pessoas surdas vivenciados por familiares ouvintes: estudo exploratório.

Online braz. j. nurs. (Online). [Internet], 2020 [cited 2020 Jun 28]; 18(3). DOI: https://doi.org/10.17665/1676-4285.20196212

12. Santos A, Portes AJF. Perceptions of deaf subjects about communication in Primary Health Care. Rev. Latino-Am. Enfermagem [Internet], 2019 [cited 2020 Jan 26]; 27( e3127). DOI: http://dx.doi.org/10.1590/1518-8345.2612.3127

13. Marquete VF, Costa MAR, Teston EF. Communication with hearing impaired people from the perspective of health professionals. Rev. baiana enferm. [Internet], 2018 [cited 2019 Sep 12]; 32: e24055. DOI: http://dx.doi.org/10.18471/rbe.v32.24055

14. Chirney L, Rodrigues AL. Survey and analysis of spatial organizations deriving from the metropolization process of the Region of Maringá. Cad. Metróp. [Internet], 2020 [cited 2020 Jun 05]; 22(47):173-92. DOI: http://dx.doi.org/10.1590/2236-9996.20204708

15. Goodman L. Snow ball sampling. Annals of Mathematical Statistics [Internet], 1961 [cited 2018 Jun 10]; 32(1):148-70. DOI: https://doi.org/10.1214/aoms/1177705148

16. Spreen M. Rare Populations, Hidden Populations, and Link-Tracing Designs: What and Why? Bull Methodol Sociol [Internet], 1992 [cited 2018 Jun 10]; 36(1):34-58. DOI: https://doi.org/10.1177/075910639203600103

17. Driessnack M. Who Are You From?": The Importance of Family Stories. J. Fam. Nurs. [Internet], 2017 [cited 2020 Mar 15]; 23(4):434-49. DOI: https://doi.org/10.1177/1074840717735510

18. Anderson ML, Wolf Craig KS, Hall WC, Ziedonis DM. A Pilot Study of Deaf Trauma Survivors' Experiences: Early Traumas Unique to Being Deaf in a Hearing World. J Child Adolesc Trauma [Internet], 2016 [cited 2020 Mar 20]; 9(4):353-8. DOI: https://doi.org/10.1007/s40653-016-0111-2

19. Fleshner M, Crane CR. Exosomes, DAMPs and miRNA: Features of Stress Physiology and Immune Homeostasis. Trends Immunol [Internet], 2017 [cited 2020 Apr 17]; 38(10):768-76. DOI: https://doi.org/10.1016/j.it.2017.08.002

20. Grace PM, Hutchinson MR, Maier SF, Watkins LR. Pathological pain and the neuroimmune interface. Nat. Rev. Immunol. [Internet], 2014 [cited 2020 Apr 17]; 14(4):217-31. DOI: https://doi.org/10.1038/nri3621

21. Hall WC, Smith SR, Sutter EJ, DeWindt LA, Dve TDV. Considering parental hearing status as a social determinant of deaf population health: Insights from experiences of the "dinner table syndrome". PloS One [Internet], 2018 [cited 2020 Mar 30]; 13(9):e0202169. DOI: https://doi.org/10.1371/journal.pone.0202169

22. Smith SR, Samar VJ. Dimensions of Deaf/Hard-of-Hearing and Hearing Adolescents' Health Literacy and Health Knowledge. J. Health Commun. [Internet], 2016 [cited 2020 Apr 15]; 21(sup2): 141-54. DOI: https://doi.org/10.1080/10810730.2016.1179368

23. Santos PJP. The family and its commitment to education: your participation in child education. Revista Educação e Ciências Sociais [Internet], 2019 [cited 2020 Aug 25]; 2(3):92-112. DOI: https://doi.org/10.38090/recs.2595-9980.2019.v2.n3.92-112

24. Haines J, Rifas-Shiman SL, Horton NJ, Kleinman K, Bauer KW, Davison KK, et al. Family functioning and quality of parentadolescent relationship: cross-sectional associations with adolescent weight-related behaviors and weight status. Int. J. Behav. Nutr. Phys. Act. [Internet], 2016 [cited 2020 Apr 19]; 13 (68). DOI: https://doi.org/10.1186/s12966-016-0393-7

25. Rufino EC, Andrade SSDC, Leadebal ODCP, Brito KKG, Santos SSH. Women's knowledge about sti/aids: working with health education. Ciênc. Cuid. Saúde [Internet], 2016 [cited 2020 Mar 10]; 15(1):9-16. DOI: https://doi.org/10.4025/cienccuidsaude.v15i2.26287 